\title{
Constraints on access in a problem solving context
}

\author{
GREG A. PERFETTO, JOHN D. BRANSFORD, and JEFFERY J. FRANKS \\ Vanderbilt University, Nashville, Tennessee 37240
}

\begin{abstract}
The effects of previously acquired information on a later problem solving task were explored. Prior research has shown that the acquisition of potentially relevant information is not effective for cuing solutions in a later problem solving task unless subjects are informed of the connection. The present research extends these results and demonstrates that the problem solving failure is not due to subjects' rejecting the potentially relevant information following retrieval. Rather, the apparent failure to appropriately use previous information is a result of uninformed subjects' inability to spontaneously access such information. Furthermore, the observed access failure is not reversible by simply informing the subjects of the task connection prior to a second trial. Finally, the results indicate that problem solving failure on a later informed trial is a problem-specific phenomenon that does not generalize to new problems. The implications for contemporary episodic memory paradigms and the role of access in learning theory are discussed.
\end{abstract}

Effective comprehension and problem solving require more than the availability of potentially relevant information; people must access this information when it is needed (e.g., Bransford \& Johnson, 1972, Experiment 2). One method for investigating the processes involved in accessing information is to first present subjects with potentially relevant information and to then explore the conditions necessary for them to utilize this information in problem solving tasks (e.g., Weisberg, DiCamillo, \& Phillips, 1978).

Weisberg et al. (1978) had subjects learn a list of paired associates, one of which was candle-box. It was expected that this association would later cue a solution to the "candle problem" (Dunker, 1945). The candle problem involves attaching a candle to the wall so that it will burn properly. The available materials are a box of nails, a book of matches, a hammer, and the candle. The solution sought by Weisberg et al. was to attach the box to the wall using the hammer and nails, and to then put the candle on top of or in the box. The critical manipulation was whether subjects were informed that one of the paired associates acquired previously (i.e., candlebox) was relevant to the candle problem.

Weisberg et al.'s (1978) results indicate that the candle-box association was effective in cuing the expected solution only when subjects were explicitly informed that previously acquired information was relevant to the candle problem. Weisberg et al. interpreted their results

This research was supported in part by the National Institute of Education (Grant NIE-G-79-0017). The authors wish to thank Jonathan Doner, Karen Mezynski, and Pam Auble for their helpful comments throughout the course of this research. Requests for reprints should be addressed to Greg A. Perfetto, Department of Psychology, Vanderbilt University, 134 Wesley Hall, Nashville, Tennessee 37240 . as evidence that transfer is a nonautomatic process. However, the failure of uninformed subjects to solve the candle problem can be accounted for by two distinct hypotheses. First, subjects may have spontaneously retrieved the appropriate cue when working on the candle problem but discarded it as irrelevant (note that the cue "candle-box" does not communicate much specific information). Alternately, subjects may have failed to retrieve the appropriate cue. Although both of the hypotheses provide plausible explanations for the failure of uninformed subjects to utilize prior information, they do so in very different ways.

The first hypothesis argues that subjects may have spontaneously retrieved "candle-box," but that this information may have been insufficient to facilitate problem solving because subjects did not perceive the information to be relevant. Since the information conveyed by the clue "candle-box" is not very explicit, it would not be surprising if subjects who were not informed that this information was useful failed to use it to solve the candle problem. In contrast, subjects who were informed of the connection between the tasks would expect the clue to provide relevant information and hence might be more likely to realize the significance of this information. This hypothesis implies that if clues were constructed that were highly related to problems (i.e., that provided obvious solutions), subjects should be able to utilize this information to solve problems even if the connection between the tasks was not explicitly pointed out. The second hypothesis noted above suggests that subjects actually fail to retrieve the relevant information in the absence of explicit instructions to do so. If this is the case, then increases in the relevance of the clues may still be insufficient to facilitate problem solving by uninformed subjects. 
The first experiment was designed to investigate the former hypothesis by constructing obvious clues that were relevant to problem solving. The problems chosen for this experiment were 12 "insight" problems adapted from Gardner (1978). For each problem, a sentence clue was constructed that blatantly suggested the solution to the problem. For example, one problem was "A man who lived in a small town in the U.S. married 20 different women of the same town. All are still living and he has never divorced one of them. Yet, he has broken no law. Can you explain?" The sentence constructed as a clue to this problem's solutions was "A minister marries several people each week." The relevance of the sentence clues to the problems was verified by presenting 15 pilot subjects with each problem and its respective clue sentence. In addition, the candle problem was presented to subjects with the association "candle-box." The pilot subjects were given approximately $40 \mathrm{sec}$ to solve each problem.

Overall, subjects solved an average of $96 \%$ of the insight problems. The candle problem was solved by only $46 \%$ of the subjects. Subjects were also asked to rate the clues according to the obviousness with which they pointed to a solution. The sentence clues had an average rating of 1.6 ( 1 being extremely obvious to 5 being not at all obvious), whereas the candle-box association had an average rating of 2.5. An additional analysis indicated that each of the 12 problem-sentence clue pairs was solved by more subjects than was the candle problem. Furthermore, each of the sentence clues was rated as providing a more obvious solution than the "candle-box" clue. Thus, the sentence clues constructed for the present study appear to meet the requirement of indicating an obvious solution to their corresponding problems.

\section{EXPERIMENT 1}

In Experiment 1, all experimental subjects were exposed to the sentence clues. The initial task required subjects to rate the clues on a scale of general truthfulness; this constituted an incidental acquisition. Subjects then received the set of problems. One group of subjects was explicitly told that the sentences they had rated would help them solve the problems they were about to receive. No mention of this connection was made to a second group. A control group did not receive the acquisition sentence clues prior to their problem solving attempts.

\section{Method}

Subjects. Subjects were 60 undergraduate students enrolled in an introductory psychology class at Vanderbilt University. They received course credit for their participation in the experiment.

Materials and Procedure. Materials for this experiment included the 12 problem-sentence clue pairs described previously. Subjects were introduced to the experiment by the experimenter, who told them that they would be given several tasks to complete. The subjects were told that the purpose of these tasks was to gather normative data for future experiments.

Subjects were first asked to rate 14 statements on a 5-point scale of truthfulness (e.g., never true, sometimes true, always true). Twelve of the 14 statements were the sentences constructed to clue solutions to the experimental problems. The first and last statements were fillers that were similar in form to the other statements but unrelated to the experimental problems. Subjects were allowed $20 \mathrm{sec}$ to read and rate each statement. This rating task constituted an incidental acquisition for the subsequent problem solving task.

Following the acquisition task, a delay of $3 \mathrm{~min}$ was provided by collecting the rating sheet and directing subjects to fill out their attendance forms. All subjects were then told that they were going to receive some problems to solve. Subjects in the first group were told that the sentences they had just rated would help them to answer most of the problems (informed group). A second group was not made aware of the connection between the tasks (uninformed group). Both groups were then given problem booklets and answer sheets. The problem booklets contained one of four randomized orders of the experimental problems. In addition, prior to the 12 experimental problems, all of the problem booklets included 3 additional problems taken from Gardner (1978). These three initial filler problems were included to reinforce the separation of the acquisition and problem solving tasks, and to provide an additional delay (for a total of $5 \mathrm{~min}$ ) between the end of the acquisition task and the first clue-related problem. Subjects were allowed $40 \mathrm{sec}$ to read each problem and write down a solution. They were not allowed to turn the page to the next problem until the full $40 \mathrm{sec}$ had expired. A third group (baseline) did not receive the acquisition (clue) sentences. Instead, these subjects were immediately given the problems to solve.

After the problem solving task, all subjects were asked to fill out a questionnaire. One purpose of the questionnaire was to assess the degree to which informed and uninformed subjects were aware that a relationship existed between the acquisition task and the problem solving task. Subjects were also asked to indicate which, if any, problems they had seen prior to the experiment and to answer questions about the processes they used during their problem solving attempts.

\section{Results}

Subjects who had previously seen more than two of the experimental problems were dropped from the study. By this criterion, data from three subjects in each of the experimental groups (informed and uninformed) and from four subjects in the baseline group were not used in the analyses. In addition, four subjects in the uninformed condition reported that they were aware of the purpose of the experiment before they received any of the problems. Because they were not actually uninformed, data from these subjects were not used in the analyses. Additional subjects were tested to attain 20 subjects in each condition.

The primary data involved subjects' answers to the 12 problems. A preliminary analysis revealed that all subjects generated some sort of answer to each problem, but that many answers were inadequate. For example, the problem "Uriah Fuller, the famous Israeli superpsychic, can tell you the score of any baseball game before the game starts. What is his secret?" was of ten answered by "He is a superpsychic." (The sentence clue was "Before any game is played, there is no score.") Similarly, the 
problem "Why are 1977 dollar bills worth more than 1976 dollar bills?" was often answered by a reference to inflation, whereas the actual clue was "l,966 dollar bills are worth more than a 1967 dollar bill."

The mean proportion of solutions that were congruent with the clue sentences (these clues suggest the only appropriate answers that we have found for our problems, i.e., those that do not violate constraints on the problem) were $.19(\mathrm{SD}=.118), .29(\mathrm{SD}=.216)$, and $.54(\mathrm{SD}=.231)$ for subjects in the baseline, uninformed, and informed groups, respectively. A one-way ANOVA revealed a significant main effect $[F(2,57)=16.80$, $\mathrm{p}<.001]$. Pairwise comparisons using Dunn's procedure (Kirk, 1968) were performed to investigate the relative performance of each group. Subjects in the uninformed group were not superior to those in the baseline condition $\left[t^{\prime} D(3,57)=1.57, p>.05\right]$. In contrast, subjects in the informed condition produced more correct solutions than both baseline subjects $\left[t^{\prime} D(3,57)=5.62, p<.01\right]$ and uninformed subjects $\left[t^{\prime} D(3,57)=4.06, p<.01\right]$.

An item analysis of the number of correct responses made by each group to each problem was also performed in order to determine if the observed data were consistent across the different problems or if the results might be due to the effects of only a few problems. For each of the 12 problems, subjects in the informed group produced more solutions than did subjects in either the uninformed or baseline groups $(\mathrm{N}=12, \mathrm{P}=12, \mathrm{Q}=0$; $p<.001)$. A comparison of the uninformed and baseline groups revealed that uninformed subjects produced more solutions to only 8 of the 12 problems; this was not significant by a sign test.

Subjects' responses to the questionnaire revealed two main results. First, subjects in the uninformed group reported that it did not occur to them to try to use the clues presented during acquisition when attempting to solve the problems. They therefore did not appear to spontaneously access these clues and then reject them because the clues seemed to be irrelevant to the problems they were trying to solve. Second, the comments of subjects in the informed group seemed to indicate that problems tended either to cue the appropriate acquisition clue or to cue no specific acquisition information. None of the subjects in the informed group indicated that they attempted to free recall the acquisition clues until they found one that helped them solve a particular problem. Instead, the problems frequently acted as retrieval cues that permitted access to the relevant clue.

\section{Discussion}

The results of the first experiment replicate those reported by Weisberg et al. (1978). In the present study, subjects who were not explicitly told that the sentences they had rated were useful problem solving clues did not use this information when presented with the experimental problems. In contrast, the identical clues were used by subjects in the informed group. Due to the nature of the sentence clues, it is unlikely that subjects would have failed to solve a problem had they retrieved the relevant clue. Furthermore, subjects' reports indicated that they did not attempt to recall the clue information while uninformed. Thus, the uninformed subjects' problem solving failure does not appear to be due to their actually retrieving the appropriate clues but then failing to recognize the relevance of these clues to problem solutions.

Our initial belief before conducting Experiment 1 was that even subjects in our uninformed condition would spontaneously access the relevant acquisition clues because the latter seemed so obviously relevant to the problems that the subjects were asked to solve. However, the results of Experiment 1 indicate that subjects in fact failed to spontaneously access obviously relevant information unless they were explicitly informed of the relationship between the acquisition task and the problemsolving task. The use of informed and uninformed conditions therefore seems to be an important manipulation for exploring the conditions necessary for access to occur.

The results of Experiment 1 provide an additional opportunity to explore possible constraints on the processes involved in accessing relevant information. As we noted earlier, subjects in all conditions (baseline, uninformed, informed) generated some sort of answer to the problems they were asked to solve. The present design therefore allows us to explore the consequences of producing an inappropriate answer to a problem. In particular, what if subjects who are initially uninformed are given a second trial in which they are informed that their acquisition experiences are relevant to the problems they are trying to solve? Will they now be able to access relevant information or will they be hurt by the fact that they initially. generated answers that were incorrect? This issue is explored in Experiment 2.

\section{EXPERIMENT 2}

\begin{abstract}
Method
Subjects. Subjects were 60 undergraduate students enrolled in an introductory psychology class at Vanderbilt University. They received course credit for their participation in the experiment.

Materials and Procedure. Materials for Experiment 2 were the same as those used in Experiment 1. However, unlike Experiment 1 , subjects were given two attempts to solve the problems (Trial 1 and Trial 2). The procedures for the first trial were the same as those described in the first experiment. That is, informed and uninformed groups were first given the acquisition rating sheet. After completion of the acquisition task, the relationship between the rated clues and the problems was pointed out to subjects in the informed condition. Uninformed subjects did not receive these instructions. Both groups were then given the problems to solve. Subjects in the baseline condition solved the problems without having seen the acquisition items. All subjects then completed a questionnaire. These procedures constituted a replication of Experiment 1 . Following this, both informed and uninformed subjects were told of the connection between the acquisition items and the problems.
\end{abstract}


In order to make the connection between acquisition and problem solving clear, subjects were given an example. This example consisted of the experimenter reading the marriage problem and its corresponding clue from the rating sheet. This illustration problem was the same for all subjects and was not included in the Trial 2 analyses conducted on the remaining 11 problems. Subjects were then administered a second trial on all problems. This was done in the same manner as Trial 1 , with subjects being given $40 \mathrm{sec}$ to answer each problem.

\section{Results}

Subjects who had previously seen more than two of the experimental problems were dropped from the study. By this criterion, data from four subjects in each of the experimental groups (informed and uninformed) and three subjects in the baseline group were not included in the analyses. In addition, three subjects in the uninformed condition reported that they were aware of the purpose of the experiment before they received any of the problems. Because they were not actually uninformed, data from these subjects were not used in the analyses. Additional subjects were tested to attain 20 subjects in each condition.

Since one of the problems and clues was used as an example prior to Trial 2, data from Experiment 2 were transformed into proportions prior to being analyzed. The mean percentage of correct solutions for each of the six conditions is presented in Table 1.

The data were analyzed in a 2 (trials) by 3 (time of informed) ANOVA. There were significant main effects for both trials $[F(1,57)=23.8, p<.001]$ and when subjects were informed $[F(2,57)=30.6, p<.001]$. The interaction was also significant $[\mathrm{F}(2,57)=5.1, \mathrm{p}<.01]$. Tests of simple main effects were conducted by means of Dunn's procedure in order to investigate the relative performance of subjects on Trials 1 and 2. The Trial 1 data replicated Experiment 1 . Uninformed subjects did not solve a significantly greater proportion of problems than did baseline subjects [ $\left.\mathrm{t}^{\prime} \mathrm{D}(4,57)=1.29, \mathrm{p}>.05\right]$. However, the informed subjects produced a greater proportion of correct solutions than did those in the uninformed condition [ $\left.\mathrm{t}^{\prime} \mathrm{D}(4,57)=4.67, \mathrm{p}<.01\right]$.

The pattern of results for Trial 2 was similar. Informed subjects produced a significantly greater proportion of solutions than did subjects in the uninformed condition $\left[t^{\prime} D(4,57)=2.62, p<.05\right]$. The difference between the uninformed and the baseline groups approached but did not reach significance $\left[\mathrm{t}^{\prime} \mathrm{D}(4,57)=2.25, \mathrm{p}>.05\right]$.

\section{Discussion}

The results for the first trial of Experiment 2 repli-

Table 1

Mean Percentage and Standard Deviations of Problems Solved in Experiment 2

\begin{tabular}{lccccc}
\hline & \multicolumn{2}{c}{ Trial 1 } & & \multicolumn{2}{c}{ Trial 2 } \\
\cline { 2 - 3 } \cline { 5 - 6 } & Mean & SD & & Mean & SD \\
\hline Baseline & 17.9 & 10.6 & & 26.3 & 14.1 \\
Uninformed & 26.2 & 14.6 & & 40.9 & 19.2 \\
Informed & 56.3 & 17.1 & & 57.7 & 17.5 \\
\hline
\end{tabular}

cate those found in Experiment 1. That is, subjects in the informed group were more likely to produce correct solutions to the problems than were subjects in the uninformed or baseline groups.

The major purpose of Experiment 2 was to explore an additional question: What would happen if subjects in the uninformed group were told that their initial acquisition experiences provided information that was relevant to each problem's solution and were then given a second chance to solve the problems? The results for the second trial of Experiment 2 indicate that these subjects still perform at a level that is inferior to that of subjects in the group informed at Trial 1.

There are several possible reasons for the inferior performance of subjects who were not informed until Trial 2. One is that they may have forgotten much of the information that was presented during acquisition. Note that subjects who were initially informed on Trial 1 had the chance to activate previously experienced clues on Trial 1. This activation of relevant information could retard the forgetting process. Some sort of differential forgetting hypothesis may therefore account for the Trial 2 results found in Experiment 2.

There is a second possible reason for the inferior Trial 2 performance of subjects who were not informed until Trial 2. We noted in Experiment 1 that subjects in all groups generated some answer to each problem. The same was true in Experiment 2. It is possible that subjects who were uninformed on Trial 1 felt that most of their Trial 1 answers were correct. They may therefore have decided to provide the same answers on Trial 2. We will refer to this hypothesis as the "my answer is better than yours" hypothesis.

The purpose of Experiment 3 is to evaluate both of the hypotheses mentioned above. The differential forgetting hypothesis can be evaluated by adding an additional control group that solves different kinds of problems during Trial 1 and then becomes informed (with respect to the original set of experimental problems) on Trial 2. If subjects in this group perform as well on the second trial as those who were informed on the first trial, we can conclude that the initial clue information is still available.

The "my answer is better than yours" hypothesis can be evaluated by changing the task requirements on the second trial. In particular, subjects can be told that their task is to remember the clue sentences presented during acquisition and that they can use the problems as retrieval cues. These instructions specify a goal that should prompt subjects to attempt to remember the information provided during the acquisition task.

\section{EXPERIMENT 3}

\section{Method}

Subjects. Subjects were 60 undergraduate students enrolled in an introductory psychology class at Vanderbilt University. They received course credit for their participation in the experiment.

Procedure. The methods were identical to those used in 
Experiments 1 and 2 except for the test instructions on Trial 2. Three groups participated in the study: An informed group, an uninformed group, and an activity control group. All groups rated the acquisition sentences and were then given two trials to solve the problems.

On the first problem solving trial, the procedure for subjects in the informed and uninformed groups was identical to that of Experiments 1 and 2. Subjects in the activity control condition did not receive the experimental problems on the first trial. Instead, they received a sheet of 15 "incomprehensible" sentences (Auble, Franks, \& Soraci, 1979) to solve. They were allotted the same amount of time to solve these problems as were subjects in the informed and uninformed groups.

During the second test, all groups received the experimental problems. However, instead of being asked merely to solve the problems as on Trial 2 of Experiment 2, all subjects were asked to try to recall the initial acquisition clues and were told they could use the presented problems as retrieval cues.

\section{Results}

All subjects in this experiment met the criteria of being naive with respect to the purpose of the study and being familiar with no more than two problems. Thus, the data from all subjects were included in the analysis.

Means and standard deviations for the three groups are displayed in Table 2. The proportion of experimental problems solved correctly on Trial 1 was $.22(\mathrm{SD}=.161)$ for the uninformed group and $.65(S D=.222)$ for the informed group. (Note that the activity control group solved a different set of problems on Trial 1.) Informed subjects solved a greater proportion of problems than did uninformed subjects $[\mathrm{T}(1,38)=7.14, \mathrm{p}<.001]$, These results replicated those found in Experiments 1 and 2.

On Trial 2, all subjects were asked to recall the clue sentences presented during acquisition and were told that the problems could serve as retrieval cues. Paraphrases of the acquisition sentences were counted as correct, and the clue information did not have to be paired with the appropriate problem in order to be counted as correct (although all but three acceptable responses were correctly paired with the appropriate problems). The cued recall data for Trial 2 (see Table 2) were initially analyzed as a one-way ANOVA with three levels. The main effect was significant $[F(2,57)=28.77$, $p<.001]$. Comparisons between the three groups, calculated by Dunn's procedure, revealed that subjects in both the informed and the activity control conditions recalled a significantly greater proportion of clues than did subjects in the uninformed condition $\left[t^{\prime} \mathrm{D}(3,57)=\right.$ 5.93, $\mathrm{p}<.01$, and $\mathrm{t}^{\prime} \mathrm{D}(3,57)=7.05, \mathrm{p}<.01$, respectively]. There was no difference between the per-

Table 2

Mean Percentage and Standard Deviations of Problems Solved (Trial 1) and Cues Recalled (Trial 2) in Experiment 3

\begin{tabular}{lccccc}
\hline & \multicolumn{2}{c}{ Trial 1 } & & \multicolumn{2}{c}{ Trial 2 } \\
\cline { 2 - 3 } \cline { 5 - 6 } & Mean & SD & & Mean & SD \\
\hline Uninformed & 21.7 & 16.1 & & 38.3 & 21.0 \\
Informed & 65.4 & 22.2 & 73.8 & 20.6 \\
Control & & & 80.4 & 14.1 \\
\hline
\end{tabular}

formance of subjects in the informed and activity control conditions $\left[t^{\prime} \mathrm{D}(3,57)=1.12, \mathrm{p}>.05\right]$.

\section{Discussion}

The results of Experiment 3 rule out several possible explanations for the findings in Experiment 2. In Experiment 2, uninformed subjects received a second chance to solve the problems after being informed that the information presented during acquisition was relevant to the problems' solutions. Their performance on the second trial was nevertheless inferior to the performance of subjects who were in the informed group (informed during Trial 1). One possible explanation for this deficit was that the general act of attempting to solve problems during Trial 1 may have hindered subjects' abilities to remember the acquisition information on Trial 2. In Experiment 3, however, subjects in the activity control group also attempted to solve problems during Trial 1. Nevertheless, their performance on Trial 2 was as good as the group who had been informed on both Trial 1 and Trial 2.

Another possible explanation for the results of Experiment 2 was that subjects in the uninformed group may not have attempted to access acquisition information during Trial 2 because they were content with the answers they had produced during Trial 1. In Experiment 3, however, the Trial 2 task was changed from problem solving to one of memory for the acquisition information. Even under these conditions, subjects who were not informed until Trial 2 performed more poorly than did those in the informed and activity control groups.

There is a third possible explanation for the Trial 2 results of Experiments 2 and 3 that has not yet been evaluated: namely, that the inability of uninformed subjects to perform well on the second trial (when they become informed) may be problem specific. The act of providing an incorrect answer to a particular problem on the first trial may hamper subjects' abilities to provide a correct answer to that problem on the second trial. This hypothesis is investigated in Experiment 4 by having subjects attempt only one half of the problems on Trial 1 and then giving them all of the problems (plus informing the uninformed subjects on Trial 2). If there are constraints on access that are problem specific, we would expect the following pattern of Trial 2 data. Subjects who were not informed until Trial 2 should do as well as the initial informed group on those problems that were not presented during Trial 1 (on new problems). For old problems (those that did occur on Trial 1), however, the deficits found in Experiments 2 and 3 should still occur.

\section{EXPERIMENT 4}

\section{Method \\ Subjects. Subjects were 40 undergraduate students enrolled in an introductory psychology course at Vanderbilt University. They received course credit for their participation.}


Procedure. The design of Experiment 4 was slightly modified from that of the previous experiments. The two subject conditions (uninformed and informed) each received an equivalent acquisition, which was identical to that of the previous experiments. The procedures for the administration of Trials 1 and 2 were also the same as in prior experiments, with the following modifications. During Trial 1 problem solving, subjects attempted only 9 problems: the 3 initial fillers and 6 of the 12 experimental problems. Subjects then were given a second trial that consisted of all 15 problems: the 3 fillers, 6 old problems (attempted on Trial 1), and 6 new problems.

The materials for this experiment were constructed from the problems used in the previous experiments. On the basis of prior data, the 12 experimental problems were divided into two lists, each containing 6 problems. This was done by using the data from Experiment 2 to rank order the problems in terms of the total number of correct solutions produced. The problems that had even-numbered ranks were then grouped into List $A$ and the problems with odd-numbered ranks formed List $B$. Five random orderings of each list were then constructed and made into problem booklets, with each booklet beginning with the three foil problems. The resulting 10 booklets constituted the material for Trial 1 .

Materials for Trial 2 were constructed by making 10 random orders of all 12 problems, with the constraint that no more than two items from a given list could appear consecutively. These 10 orderings were then made into booklets, which again were prefaced with the three foil problems.

\section{Results}

Subjects who had previously seen more than two of the problems were dropped from the study. By this criterion, data from two subjects in the informed group were not used in the analyses. Additional subjects were tested to attain 20 subjects in each condition.

The data were initially analyzed for differences between Lists $A$ and $B$. There were no differences between the number of solutions produced to the lists, either overall or by subjects within the two conditions. Thus, the following analyses are collapsed across the list factor.

Data for Trials 1 and 2 were analyzed separately. Informed subjects solved more problems on Trial 1 than did uninformed subjects $[\mathrm{t}(1,38)=8.55, \mathrm{p}<.001]$. These findings replicated those reported in Experiments 1,2 , and 3 .

Trial 2 data were analyzed in a 2 (time of informed) by 2 (old-new) ANOVA, with the old-new factor being within-subjects (note that old problems are those that appeared on Trials 1 and 2, whereas new problems appeared only on Trial 2). Means are presented in Table 3.

Table 3

Mean Percentage and Standard Deviations of Problems Solved in Experiment 4

\begin{tabular}{|c|c|c|c|c|c|c|}
\hline & \multicolumn{2}{|c|}{ Trial 1} & \multicolumn{4}{|c|}{ Trial 2} \\
\hline & \multirow[b]{2}{*}{ Mean } & \multirow[b]{2}{*}{ SD } & \multicolumn{2}{|c|}{ Old } & \multicolumn{2}{|c|}{ New } \\
\hline & & & Mean & SD & Mean & $\mathrm{SD}$ \\
\hline Uninformed & 18.3 & 20.2 & 43.3 & 23.8 & 62.5 & 22.2 \\
\hline Informed & 73.3 & 20.5 & 80.8 & 16.5 & 69.2 & 23.7 \\
\hline
\end{tabular}

The old-new main effect was not significant $[F(1,38)=1.01, p>.05]$. A significant main effect was found for the time-of-informed factor $[F(1,38)=14.9$, $\mathrm{p}<.001]$. Most important, there was an interaction between the factors $[F(1,38)=17.1, p<.001]$. The source of this interaction was investigated by an analysis of simple main effects. Informed subjects produced more correct solutions to old problems (those seen on Trial 1) than to new problems $[t(1,38)=2.33, \mathrm{p}<.05]$. This pattern was reversed for the uninformed subjects, with more solutions being produced for new problems than old problems $[t(1,38)=3.29, \mathrm{p}<.01]$. The performance of informed and uninformed subjects was statistically equivalent on the new problems $[t(1,38)=$ $.917, \mathrm{p}>.05]$, but informed subjects solved more old problems $[\mathrm{t}(1,38)=5.79, \mathrm{p}<.001]$.

\section{Discussion}

In Experiments 2 and 3, uninformed subjects who received a second chance to solve the experimental problems (after being informed of the relevance of information presented duirng acquisition) still performed more poorly than did subjects who had been informed on the first trial. The results of Experiment 4 provide important information about reasons for those results. In particular, the results illustrate conditions in which the Trial 2 performance of subjects who were not informed until the second trial was as good as the performance of subjects who were informed on the first trial. These results occurred only under conditions in which subjects attempted to solve Trial 2 problems that had not been attempted during Trial 1 (i.e., under conditions when the problems were new). These data suggest that subjects who were not informed until Trial 2 did not experience a general deficit in performance due to Trial 1 problem solving (see also the results for the activity control group in Experiment 3 ). Instead, deficits in performance seemed to be problem specific; they occurred only on old problems that uninformed subjects had already tried to solve on Trial 1 .

Why were subjects who attempted Trial 1 problems while uninformed unable to access acquisition information relevant to these specific problems during Trial 2? One approach to this question is to note that these subjects actually did generate answers to these problems on Trial 1, albeit not very adequate answers; thus, these self-generated answers would be an integral part of the original encoding context for these problems. In line with the reasoning from the encoding specificity principle (Thomson \& Tulving, 1970; Tulving \& Thomson, 1973), later re-presentation of the clues during Trial 2 would be expected to result in retrieval of these previously encoded answers. Furthermore, research such as that of Bobrow and Bower (1969) and Slamecka and Graf (1978), which has demonstrated superior memory for self-generated materials, suggests that the 
tendency to retrieve these prior answers would be further enhanced because they were self-generated. Thus, it seems plausible that Trial 1 answers were retrieved during Trial 2 and that this retrieval interfered with access to the information presented during the acquisition task. The present design does not allow us to state whether subjects must actually generate an inappropriate answer on Trial 1 in order to show a decrement for old items on Trial 2 problem solving. Additional experiments must be designed in order to address this question. For present purposes, the important point is that initial failures to access potentially relevant information (e.g., during Trial 1) can cause problem-specific deficits that are not easily eradicated simply by becoming informed.

\section{GENERAL DISCUSSION}

Two major findings emerged from the present experiments. First, the results of all four experiments indicate that subjects did not spontaneously utilize previously acquired information to solve problems unless they were explicitly informed about the relationship between initial acquisition and the later problem solving. These results replicate those found by Weisberg et al. (1978). Weisberg et al. had subjects learn associations such as "candle-box" and demonstrated that this did not help them solve Dunker's (1945) candle problem unless they were explicitly informed about the relationship between acquisition and test. We argued that the clue "candlebox" was not particularly informative and hence devised materials in which the clues provided information that was more likely to lead to problem solution (see the initial assessment of clue effectiveness described prior to Experiment 1). Even under these conditions, the present results show that subjects who were informed about the acquisition-test relationship were much more likely to utilize the acquisition information than were subjects who were not informed about this relationship. Furthermore, uninformed subjects did not solve a significantly greater number of problems than did subjects in the baseline groups.

The results discussed above are also consistent with investigations by Gick and Holyoak (1980) of the ability to transfer solutions between analogical problems. Gick and Holyoak found that subjects typically failed to transfer a solution read in conjunction with one problem to an analogous problem (Dunker's 1945, "radiation problem") unless they were given a hint to use the prior story to help them solve the problem. However, Gick and Holyoak's task required an abstract matching of the target problem and solution to the analogous problem. The direct nature of the materials in the present experiment demonstrates that this phenomenon is not the result of subjects' inability to "see the analogy" but is of a more general nature. Furthermore, neither Weisberg et al. (1978) nor Gick and Holyoak consider the effects attributable to the initial spontaneous transfer failure. These effects are discussed below.

A second major result of the present experiments involves the consequences of initial failures to access potentially relevant information. In Experiment 2, for example, uninformed subjects were not told that their acquisition experiences were relevant to the problems they were about to solve on Trial 1 and hence performed more poorly than informed subjects. However, even when uninformed subjects were informed on Trial 2, they were still less likely to use relevant acquisition information to generate solutions than were subjects in the informed group. The results of Experiments 3 and 4 indicated that this decrement was not due to the general act of attempting to solve problems during Trial 1. Instead, the decrement occurred only for Trial 2 problems that had actually been attempted during Trial 1. Thus, initial failures to access relevant information can lead to problem-specific deficits in later problem solving performance. As discussed previously, these deficits can be seen as resulting from retrieval processes operating under constraints due to encoding specificity and possibly to the self-generated nature of the answers encoded during Trial 1 ,

The present results are similar to findings of research conducted in an educational setting. For example, Howe (1970) found that the contents of weekly attempts at written reproduction were very closely related to previous recall attempts. The contents of a given recall attempt, even when incorrect, were more likely to recur in the succeeding weeks than were correct but non recalled items. This was true despite an intervening presentation of the correct material.

The importance of the present findings can be seen when one notes that many, if not most, ordinary problem solving situations involve cases in which the problem solver is essentially uninformed and is engaged in self-generation of potential answers to the problem. To have any chance of solving such problems, one must presume that the problem solver has previously acquired infomation that is relevant to the problem solution. A major aspect of problem solving involves accessing this relevant information. However, usually the problem solver does not have someone telling him what information is relevant or when it was acquired (i.e., the problem solver is uninformed). The present work indicates that this uninformed state can lead to serious difficulties in accessing previously acquired, relevant information. Furthermore, in attempting to solve problems in this uninformed state, the problem solver will probably generate at least partial answers, which are inadequate solutions to the problem. The present work indicates that these inadequate self-generated answers may lead to even greater deficits in accessing relevant information and problem solving in any future attempts to solve the same or similar problems. Given such possible difficulties, one important area for future research involves 
investigations of types of acquisition experiences (and/or kinds of access or retrieval strategies) that can be engaged during problem solving. Specifically, researchers might investigate the types of experiences that are effective in overcoming deficits that stem from being uninformed as to when the relevant information was acquired.

Although the present work was primarily concerned with problem solving, the present findings also may have implications for the study of a broad range of psychological phenomena. For example, the present results suggest that some traditional experimental paradigms may lead to an overestimation of the accessibility of relevant information. Consider, for example, typical episodic memory experiments. In such experiments, subjects are presented with acquisition information and are then asked to remember this information, perhaps with the help of various retrieval cues. Of necessity in such experiments, subjects are "informed" concerning the relationship between the acquisition and test. Given the present findings, a major question concerns the generalization of the findings from such typical memory experiments to cases of more spontaneous remembering or recollection (i.e., to cases in which the rememberer is essentially uninformed). It seems obvious that much remembering, in ordinary circumstances, involves such uninformed spontaneity. Furthermore, even in circumstances in which one is asked to remember some particular information, the asker often does not or cannot inform the rememberer about where the rememberer acquired the relevant information. Both of these circum. stances differ from the procedures involved in typical memory studies. In general, many typical experimental paradigms prompt access of information under explicitly informed conditions. The present findings suggest that such studies may often seriously underestimate the potential problems involved in accessing such information.

Finally, the finding that access to relevant information is strongly influenced by whether one is informed or uninformed has important implications for educational practice. Many classroom tests designed to assess learning seem to be episodic in nature. That is, students know that the correct answers to problems were provided by a particular professor, set of readings, and so forth. They are therefore informed about possible sources of information that are relevant to the test question they receive. Imagine that a student performs very well on a test such as this yet, when leaving the classroom, confronts everyday problems in which information from the course would be useful. Will the student spontaneously utilize this information? The results of the present experiments suggest that the answer may well be no. It seems clear that theories of learning and instruction must address the question of how information can become accessible under conditions in which students are not explicitly informed about the particular acquisition context that is relevant to the problems they confront.

\section{REFERENCES}

Auble, P. M., Franks, J. J., \& Soraci, S. A. Effort toward comprehension: Elaboration or "aha!'? Memory \& Cognition, $1979,7,426-434$.

Bobrow, S. A., \& Bower, G. H. Comprehension and recall of sentences. Journal of Experimental Psychology, 1969, 80, 455-461.

Bransford, J. D., \& Johnson, M. K. Contextual prerequisites for understanding: Some investigations of comprehension and recall. Journal of Verbal Learning and Verbal Behavior, 1972, 11, 717-726.

Dunker, K. On problem-solving. Psychological Monographs, 1945,58, No. 270.

Gardner, M. Aha! Insight. New York: W. H. Freeman, 1978.

GICx, M. L., \& HolyonK, K. J. Analogical problem solving. Cognitive Psychology, 1980, 12, 306-355.

Howe, J. A. Repeated presentation and recall of meaningful prose. Journal of Educational Psychology, 1970, 61, 214-219.

KIRK, R. E. Experimental design: Procedures for the behavioral sciences. Belmont, Calif: Wadsworth, 1968.

Slamecka, N. J., \& Graf, P. The generation effect: Delineation of a phenomenon. Journal of Experimental Psychology: Human Learning and Memory, 1978, 4, 592-604.

Thomson, D. M., \& Tulving, E. Associative encoding and retrieval: Weak and strong cues. Journal of Experimental Psychology, 1970, 86, 255-262.

Tulving, E., \& Thomson, D. M. Encoding specificity and retrieval processes in episodic memory. Psychological Review, $1973,80,352-373$.

Weiberea, R., DiCamillo, M., \& Philuipa, D. Transferring old associations to new situations: A nonautomatic process. Journal of Verbal Learning and Verbal Behavior, 1978, 17, 219-228.

(Received for publication February 22, 1982; revision accepted August 6, 1982.) 